\title{
Statistical method on nonrandom clustering with application to somatic mutations in cancer
}

\author{
Jingjing $\mathrm{Ye}^{1 *}$, Adam Pavlicek ${ }^{2}$, Elizabeth A Lunney ${ }^{2}$, Paul A Rejto ${ }^{2}$, Chi-Hse Teng ${ }^{1,3^{*}}$
}

\begin{abstract}
Background: Human cancer is caused by the accumulation of tumor-specific mutations in oncogenes and tumor suppressors that confer a selective growth advantage to cells. As a consequence of genomic instability and high levels of proliferation, many passenger mutations that do not contribute to the cancer phenotype arise alongside mutations that drive oncogenesis. While several approaches have been developed to separate driver mutations from passengers, few approaches can specifically identify activating driver mutations in oncogenes, which are more amenable for pharmacological intervention.

Results: We propose a new statistical method for detecting activating mutations in cancer by identifying nonrandom clusters of amino acid mutations in protein sequences. A probability model is derived using order statistics assuming that the location of amino acid mutations on a protein follows a uniform distribution. Our statistical measure is the differences between pair-wise order statistics, which is equivalent to the size of an amino acid mutation cluster, and the probabilities are derived from exact and approximate distributions of the statistical measure. Using data in the Catalog of Somatic Mutations in Cancer (COSMIC) database, we have demonstrated that our method detects well-known clusters of activating mutations in KRAS, BRAF, PI3K, and $\beta$-catenin. The method can also identify new cancer targets as well as gain-of-function mutations in tumor suppressors.
\end{abstract}

Conclusions: Our proposed method is useful to discover activating driver mutations in cancer by identifying nonrandom clusters of somatic amino acid mutations in protein sequences.

\section{Background}

Cancer is a genetic disease caused by the accumulation of tumor-specific (somatic) mutations in two broadly defined types of genes called tumor suppressors and oncogenes (Vogelstein and Kinzler (2004) [1]). In general, mutations in tumor suppressors tend to inactivate these natural repressors of tumorgenesis. Mutations in cellular proto-oncogenes, on the other hand, typically increase or deregulate the activity of their protein products. The existence of different types of genes and mutations in cancer has significant practical implications for developing targeted therapies in cancer care. So far, pharmacological restoration of tumor suppression function has been challenging: better success has been achieved by inhibiting activated oncogenes (Weinstein and Joe (2006) [2]). In addition to representing potential therapeutic targets, activating mutations can also be

\footnotetext{
* Correspondence: Jingjing.Ye@pfizer.com; Chi-Hse.Teng@amylin.com ${ }^{1}$ Global Pre-Clinical Statistics, Pfizer Global Research and Development, 10777 Science Center Drive, San Diego, CA, 92121, USA
}

used as biomarkers to identify populations likely to respond to therapies targeting the mutated genes. There is therefore significant interest in identifying those mutations necessary for the cancer phenotype (also known as driver mutations), separating the driver mutations from the random (passenger) mutations that occur as a consequence of the genetic instability typical for human tumors (Cahill et al. (1999) [3]), and furthermore distinguishing activating mutations from inactivating mutations.

Several methods have been developed for the automated prediction of driver oncogenic mutations in individual genes, yet few are suitable for detecting activating mutations. The most straightforward method predicts that driver mutations have a large number of mutations relative to the estimated background mutational rate, after normalizing for gene size (Wang et al. (2002) [4]; see also the "Standard test" in supplementary information for Ding et al. (2008) [5]). Another popular approach predicts that driver mutations have a higher

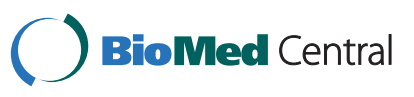

(C) 2010 Ye et al; licensee BioMed Central Ltd. This is an Open Access article distributed under the terms of the Creative Commons Attribution License (http://creativecommons.org/licenses/by/2.0), which permits unrestricted use, distribution, and reproduction in any medium, provided the original work is properly cited. 
frequency of non-synonymous mutations relative to the background mutation rate (e.g. Bardelli et al. (2003) [6]; Yang et al. (2003) [7]; Samuels et al. (2004) [8]; Davies et al. (2005) [9]; Greenman et al. (2006) [10]; Sjöblom et al. (2006) [11]). These methods are typically used to estimate the total fraction of driver mutations or to detect driver genes, but like predictions based on the phylogenic conservation of protein sequences (see Kaminker et al (2007) [12] and refs. therein), they cannot distinguish between inactivating and activating mutations. In addition, these methods are less suitable to identify driver genes that have low mutation frequencies. Meanwhile, approaches that model the destabilizing effects of mutations on protein structure (see Yip et al. (2006) [13] and refs. therein) are more suitable for tumor suppressor genes. Perhaps the most reliable approach is to classify mutations based on prior knowledge from functional studies, but functional information is by definition not available for novel or poorly characterized genes.

We propose an alternative approach to detect activating mutations in oncogenes, based on the hypothesis that only a small number of specific mutations can activate a protein. To be precise, we hypothesize that a localized cluster of amion acid mutations within a protein sequence, especially in the absence of obvious mutational hotspots, is a fingerprint of selection for the oncogenic phenotype associated with activating driver mutations. Evolutionary studies demonstrate that most amino acids replacements are either neutral or incompatible with protein function (Graur and Li (2000) [14]). Thus, activating mutations should concentrate in a small subset of protein positions and domains, while passenger mutations can be distributed more evenly along the protein sequence reflecting random chance and differences in the mutability of individual DNA codons. Consistent with this hypothesis, activating somatic amino acid mutations cluster in protein kinases (e.g. Bardelli et al. (2003) [6]; Samuels. (2004) [8]; Torkamani and Schork (2008) [15]).

Several methods in the statistics literature can be applied to detect mutation clusters. For example, Naus (1965) [16] proposed a statistical test for the maximum number of points in a fixed length cluster on a line, and developed the probability and expectation. Shortly thereafter, Naus (1966) [17] compared the power of two nonrandom clustering tests on a line; one test is the maximum number of points in a fixed-length non-overlapping interval (e.g. 1 to $p, p+1$ to $2 p$, etc. for window length $\mathrm{p}$ ) and another is the maximum number of points in a fixed-length running interval (e.g. 1 to p, 2 to $\mathrm{p}+1$, etc.), which is also called scan statistics. Scan statistics were further developed and applied by Balakrishnan and Koutras (2002) [18], and Glaz and Zhang
(2006) [19] generalized the fixed-length restriction to allow variable-length intervals by determining the maximum of a series of scan statistics each with a fixed window length. While Naus's approach and scan statistics with fixed or variable window lengths are useful, a further generalization to an arbitrary number of points in the interval is more flexible and useful to identify activating driver mutations.

In this work a new statistics method is introduced that identifies nonrandom mutation clustering without specifying the number of mutations or the cluster length. The exact and approximate distribution of the statistical measure is derived and a nonrandom mutation clustering (NMC) algorithm is developed based on the measure. We confirmed the utility of this approach by detecting well-known activating mutations in KRAS, BRAF, PI3K, and $\beta$-catenin oncogenes, as well as gainof-function mutations in several tumor suppressors.

\section{Results \\ Data Description}

Data used in this study are from COSMIC (Catalog of Somatic Mutations in Cancer) database version 40 (Forbes et al (2008) [20]) via http://www.sanger.ac.uk/cosmic. To ensure compatibility with the test assumption that the location of amino acid mutations on a protein follows a uniform distribution, we limit our analysis to studies annotated as whole gene screens in COSMIC; this eliminated a great majority of COSMIC studies annotated as partial or with missing information on the full-gene screen status. Furthermore, the cluster analysis is restricted to missense mutations: nonsense and synonymous changes are excluded. We limited our search to confirmed somatic variants or mutations that were reported in other studies as somatic. Finally, we removed redundancy in mutations in cancer cell lines, since cell line mutations are often reported by several independent studies.

\section{Nonrandom clusters in cancer genes}

Using the NMC algorithm (see Methods), 12 different proteins out of 446 contain nonrandom amino acid mutation clusters with cutoff probability of less than 0.05 , with the most significant clusters listed in Table 1 (probability < 0.01). The clusters include well-known mutation hotspots in classical oncogenes such as BRAF, RAS genes, PI3K, ERBB2/Her2, and CTNNB1/ $\beta$-catenin. Interestingly, nonrandom amino acid mutation clusters were also identified in genes not considered to be classical oncogenes and even a few tumor suppressors. Interpretation of selected positive controls is described below in more detail.

\section{Mutation hotspots in classical oncogenes}

Table 2 lists the significant clusters obtained from our set of strictly selected COSMIC studies for the BRAF, 
Table 1 Genes with significant mutation clusters (Probability < 0.01)

\begin{tabular}{lcccc}
\hline Gene & $\begin{array}{c}\text { Cluster } \\
\text { size }\end{array}$ & $\begin{array}{c}\text { Cluster } \\
\text { positions }\end{array}$ & $\begin{array}{c}\text { Number of } \\
\text { mutations in } \\
\text { cluster }\end{array}$ & $\begin{array}{c}\text { Cumulative } \\
\text { cluster } \\
\text { probability* }\end{array}$ \\
\hline KRAS (188 aa) & 2 & $12-13$ & 131 & $1.47 \mathrm{E}-234$ \\
\hline BRAF $(766$ aa) & 1 & $600-600$ & 60 & $2.02 \mathrm{E}-157$ \\
\hline TP53 (393 aa) & 155 & $132-286$ & 326 & $3.07 \mathrm{E}-101$ \\
\hline NRAS (189 aa) & 1 & $61-61$ & 33 & $7.11 \mathrm{E}-62$ \\
\hline PIK3CA (1068 aa) & 5 & $542-546$ & 27 & $7.09 \mathrm{E}-46$ \\
\hline CTNNB1 (781 aa) & 13 & $33-45$ & 12 & $8.54 \mathrm{E}-19$ \\
\hline ERBB2 (1255 aa) & 1 & $776-776$ & 2 & $7.97 \mathrm{e}-4$ \\
\hline HRAS (189 aa) & 1 & $61-61$ & 4 & $2.06 \mathrm{E}-06$ \\
\hline PTEN (403 aa) & 63 & $111-173$ & 8 & $5.50 \mathrm{E}-05$ \\
\hline MAP2K7 (419 aa) & 1 & $162-162$ & 2 & 0.002386 \\
\hline LRRK2 (2534 aa) & 4 & $1723-1726$ & 2 & 0.003547 \\
\hline *: only most significant cluster per gene is listed & &
\end{tabular}

KRAS, CTNNB1/ $\beta$-catenin, PI3K, and ERBB2 oncogenes. As expected, the most significant hotspot in BRAF was amino acid residue 600 and represents the well-known, highly oncogenic V600E mutation (Davies et al. (2002) [21]). Similarly, RAS residues 12, 13, and 61 are known to be frequently mutated in tumors (see Bos (1988) [22] for review). Residues 33, 34, 37, 41, and 45 were identified as a significant cluster in CTNNB1/ $\beta$ catenin. There is a clear mechanistic rationale: residues 33, 37 and 41 are phosphorylated directly by GSK-3 $\beta$ while residue 45 has been reported to be a primer site that is phosphorylated by CK1 (Hagen and Vidal-Puig (2002) [23]): mutations at these positions prevent GSK-3 $\beta$-mediated degradation of $\beta$-catenin (Morin et al. (1997) [24]). Residues 542-546 surround the 545 hotspot in the helical domain of PI3K/PIK3CA (Samuels et al. (2004) [8]), with a second significant PI3K cluster in the kinase domain (positions 1025-1049; probability 2.60E20; Figure 1). Mutation of Glycine 766 in ERBB2 has

Table 2 Mutation positions for selected oncogenes

\begin{tabular}{ll}
\hline Gene & Position (\# of mutations) \\
\hline BRAF(766 aa) & $464(1), 466(2), 469(4), 581(1)$, \\
& $596(2), 597(2), 600(60), 601(2)$ \\
\hline KRAS(188 aa) & $12(99), 13(32), 22(1), 23(1), 61(6)$, \\
& $117(1), 146(10)$ \\
\hline CTNNB1 (781 aa) & $6(1), 33(3), 34(2), 37(3), 41(2), 45(2)$ \\
\hline PIK3CA(1068 aa) & $88(3), 111(3), 118(1), 124(1), 345(1)$, \\
& $449(1), 453(1), 539(1), 542(5)$, \\
& $545(20), 546(2), 549(1), 1023(1)$, \\
& $1025(1), 1047(21), 1049(1), 1066(1)$ \\
\hline
\end{tabular}

The number of mutations for each position is shown in parenthesis, positions within clusters from Table 1 are highlighted in bold, and CpG positions are underlined. been reported to dramatically increase protein kinase activity (Fan et al. (2008) [25]).

For most genes in Table 1, multiple significant amino acid clusters were found, with the most significant clusters in sites of well-known oncogenic mutations. Interestingly, the great majority of the mutation-hotspots are not in CpG positions (Table 2) suggesting that selection and not the underlying mutation rate drives these changes in tumors.

\section{General remarks on detected mutation hotspots}

In addition to known clusters of activating mutations in major oncogenes, several other genes have significant mutation hot-spots. For example, two mutations between the Roc (Ras of complex proteins) and kinase domains in the LRRK2 locus form a significant cluster. The LRRK2 kinase, also known as PARK8, is not considered to be a classical cancer gene. It most closely resembles the family of tyrosine-like kinases that phosphorylate serine/threonine residues and lies upstream of mitogen-activated protein kinase (MAPK) pathways (Mata et al. (2006) [26]). Interestingly, germline polymorphisms in LRRK2 predispose affected individuals to Parkinson disease and are linked to specific cancer types (Inzelberg and Jankovic (2007) [27]; Strongosky et al. (2008) [28]).

As expected, we found fewer significant mutation hotspots in tumor suppressors, and these hot-spots were typically much larger than those associated with oncogenes. In general, inactivating amino acid mutations are not expected to form localized nonrandom clusters, but rather to span many residues in highly conserved regions (e.g. Nigro et al. (1989) [29]). The most significant cluster identified in TP53/p53 spans residues 132 286 , one of the four major p53 mutation hotspots that are highly conserved in vertebrates (Nigro et al. (1989) [29]). It overlaps the original major hotspot in residues 110-307 identified by Hollstein et al. (1991) [30], and spans two shorter hotspots that include gain-of-function mutations in positions 248 and 273 (Song et al. (2007) [31]). Structural analysis demonstrates that both regions are close together in the folded protein (Figure 2). The cluster found in the phosphatase and tensin-homology domains of PTEN includes residues known to inhibit PTEN phosphatase activity (Tolkacheva and Chan (2000) [32]), and sequence conservation cannot explain this clustering since most of the PTEN protein is well conserved among vertebrates (Yu et al. (2001) [33]). These examples demonstrate that tumor suppressor activity can be muted by changes in protein function in addition to gene deletions or disruption of the reading frame. 


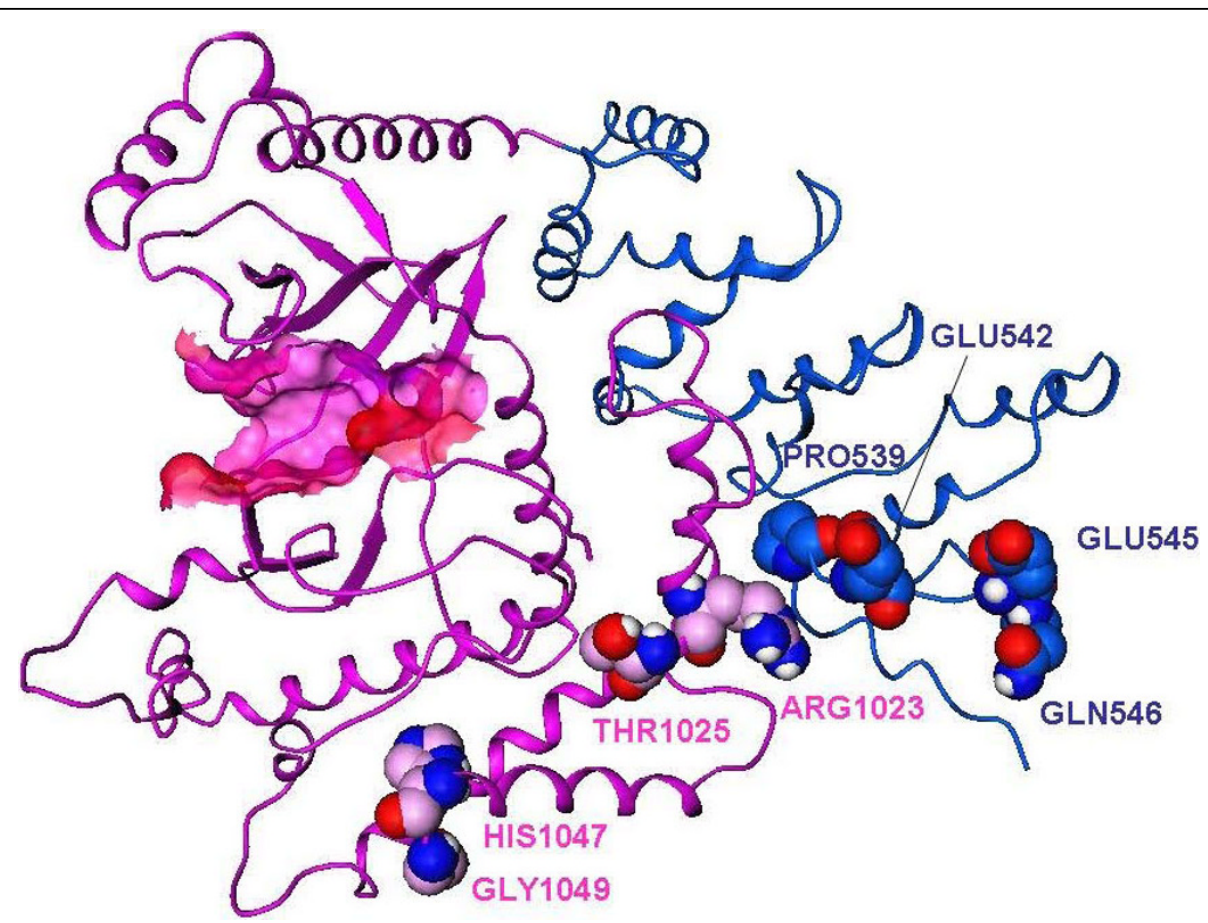

Figure 1 Ribbon representation of the PI3K $\alpha$. Ribbon representation of the PI3K $\alpha$ helical domain (blue) and kinase domain (magenta) extracted from the p110 $\alpha /$ p85 $\alpha$ complex (PDB Code: 2RD0; Berman et al. (2000) [45]; Huang et al. (2007) [46]). Displayed in CPK representations are sites of major oncogenic mutations: Pro539, Glu542, Glu545 and Gln546 in the helical domain (blue); Arg1023, Thr1025, His1047 and Gly1049 in the kinase domain (pink). The ATP binding site in the kinase domain is highlighted with a surface.

\section{Discussion and Conclusions}

A new method for the identification of nonrandom mutation clusters in biological sequences is presented. The method is fast, robust, and unlike many previous methods, it is does not require a fixed window length, which enables the identification of significant clusters of variable sizes, particularly important for the detection of activating mutations. We have applied this method to investigate somatic amino acid mutations in the COSMIC database. Our method detected very short clusters spanning a few individual amino acid positions in the case of the oncogenes BRAF or KRAS, as well as larger regions in the tumor suppressors p53 and PTEN.

A recent paper by Wagner (2007) [34] proposed two similar approaches using the distance between mutation positions. In the first approach, a Poisson distribution was utilized to model mutation clusters. The test on the distance of mutation positions containing $k$ mutations was derived and the minimum $k$ that gives significance was determined. The second approach assumed a uniform hypothesis and used permutation testing for significance. The permutation test is an approximation whose precision depends on the number of permutations undertaken, which can be very computationally intensive for good accuracy and precision. By comparison, our measure on distance is based on a uniform distribution and is calculated directly via order statistics.

Our method has several potential limitations. First of all, the status of all coding positions must be determined. This is primarily a limitation for older studies, where typically only those exons with known mutations were screened. However, with the explosion of largescale cancer genome sequencing (e.g. Sjöblom et al. (2006) [11]; Greenman et al (2007) [10]; Jones et al. (2008) [35]; Parsons et al. (2008) [36]; Cancer Genome Atlas Research Network. (2008) [37]), the number of studies suitable for analysis by NMC will grow. Another limitation is our assumption that the mutation probability is uniform: hypermutable positions for both germline and somatic mutations have been reported. As a result, we have excluded all insertions and deletions, since these mutations have strong sequence-dependence, and restricted analysis to single-point amino acid substitutions. Examples of single point mutation hotspots are $\mathrm{CpG}$ dinucleotides, which in unselected genomic sequences have more than ten-fold higher mutation frequency compared to other dinucleotides (Sved and Bird (1990) [38]). CpG hypermutability has been also reported in certain tumors (Jones et al. (1992) [39]). However, as shown in Table 2, only a few of the activating mutations identified by the NMC algorithm are in 


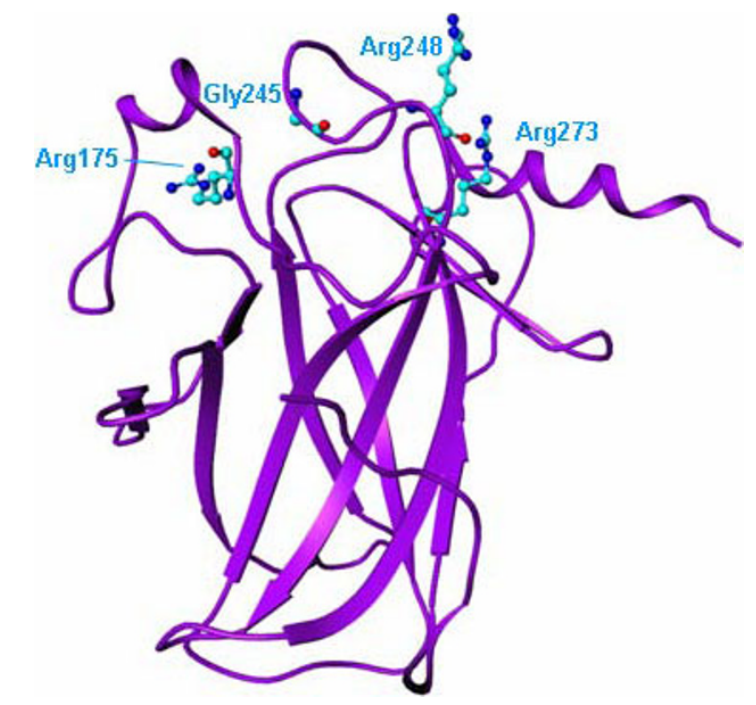

Figure 2 Ribbon representation of the human p53. Ribbon representation of the human p53 core domain X-ray structure (PDB Code: 2OCJ; Wang et al. (2007) [47]). Displayed in CPK

representation are sites of major oncogenic mutations: Arg175, Gly245, Arg248 and Arg273.

CpG sites. Another potential bias can be introduced by an unequal rate of mutagenesis caused by deficient repair of DNA damage in cells and environmental mutagens. For instance, tobacco smoke preferentially induces $\mathrm{G}$ to $\mathrm{T}$ transversions in DNA in lung cancer while colorectal tumors exhibit more transitions than transversions (Hollstein et al. (1991) [30]). Yet, despite the fact that lung and colorectal tumors have different mutational spectra, essentially all KRAS mutations in these tumors occur in residues 12, 13, and 61. In summary, while our analysis is affected by nonrandom factors such as the presence of mutation hotspots or exposure to different mutagens, positive selection for a cancer phenotype appears to be the major cause of mutation clustering.

The aim of the method is to detect activating mutations that are assumed to be concentrated in specific amino acid positions. Activating mutations are typical for cellular proto-oncogenes and, as expected, significant clusters are detected in oncogenes such as BRAF, RAS genes, CTNNB1/ $\beta$-catenin, or PI3K. Less intuitive, however, are positively selected residues in the p53 and PTEN tumor suppressors. Previous reports revealed that these genes encode functional domains that can result in gain-of-(non-suppressor)-function when altered by mutation. Thus, our method may also identify positive selection on mutations that alter the repressive function of tumor suppressors.

In conclusion, we propose a new method for discovering nonrandom clusters of mutations in biological sequences. Unlike previous approaches, the method does not use fixed length windows and therefore can be used to detect clusters of highly variable sizes. We demonstrated the value of this method to detect activating amino acid mutations in human tumors and confirmed nonrandom clustering of well-known oncogenic mutations in several classical oncogenes. The method can be also used to discover new oncogenes from largescale cancer genome data and to identify gain-of-function mutations in tumor suppressors. Finally, detection of nonrandom sequence changes is a general problem and the method may be useful in other areas such as DNA polymorphism analysis and comparative evolutionary studies (Wagner (2007) [34]).

\section{Methods}

Single amino acid mutations may lead to changes in protein function. Because missense mutations are the most likely single-point genetic mutation to have an effect on protein function, the nonrandom mutation clustering (NMC) algorithm is applied to missense mutations in individual genes in this work.

The NMC algorithm is derived under the following assumptions: 1 . each amino acid residue in a protein sequence has equal mutation probability; 2 . mutations between amino acid positions are independent; 3 . mutations between samples are independent; and 4. the number of potentially available samples is larger than the number of mutations.

Denote $N$ as the protein sequence length and $n$ as the total number of mutations in the protein. Denote $X_{i}$, a random variable between 1 and $N$, to be the position of the ith non-synonymous (missense) mutation. By assumption, the mutations follow a discrete uniform distribution, and the $n$ mutations are equivalent to $n$ independent sample draws with replacement from the discrete uniform distribution, where the probability $\mathrm{Pr}$ $\left(X_{i}=\mathrm{j}\right)=1 / \mathrm{N}$, where $\mathrm{j}=1, \ldots, N$ and $\mathrm{i}=1, \ldots, n$.

By assumption, mutations are random and can occur at the same position more than once. The data are transferred into order statistics by ordering the $X_{i}$ into $X_{(1)} \leq \ldots \leq X_{(i)} \leq \ldots \leq X_{(n)}$, where $X_{(i)}$ is the ith smallest number in the sample, $\mathrm{i}=1, \ldots, n$. To characterize clustering, the distance between order statistics $R_{k i}=X_{(k)}-X$ (i), for any pair i, k, i $<\mathrm{k}, \mathrm{i}, \mathrm{k}=1, . ., n$ is computed. We develop the distribution of $R_{k i}$, and declare the clustering to be nonrandom when the probability that the distance between order statistics $R_{k i}$ is less than a predefined significant probability level $\alpha$ : $\operatorname{Pr}\left(R_{k i} \leq r\right) \leq \alpha$. The probability $\operatorname{Pr}\left(R_{k i} \leq r\right)$ is the cumulative distribution of $R_{k i}$, the chance that the distance between order statistics $X_{(i)}$ and $X_{(k)}$ is as close or closer than $r$. Therefore, the probability $\operatorname{Pr}\left(R_{k i} \leq r\right)$ is derived as a p-value, where the probability $\alpha$ is an arbitrary level such as $0.01,0.05$, 
or 0.1 . The distance $R_{k i}$ has the simple interpretation of the size of the mutation cluster.

\subsection{Derivation of the distribution of statistical measure} While distributions of order statistics are usually derived for continuous distributions, they have also been derived for discrete distributions. Burr (1955) [40] derived the distribution of range statistics using order statistics on a discrete uniform distribution. Range statistics is a special case of our statistical measure $R_{k i}$, where $\mathrm{i}=1$ and $\mathrm{k}=$ $n$. Evans et al. (2006) [41] developed the density function and cumulative distribution of the ith order statistics given an arbitrary discrete distribution, $\mathrm{i}=1, \ldots, n$. We extend the approach of Evans et al. (2006) [41] to determine the distribution of the distance between order statistics, and generalize the approach of Burr, I.W. (1955) [40] to derive the distribution of statistics $R_{k i}$.

The distribution of $R_{k i}$ is developed from the joint distribution of order statistics $X_{(i)}$ and $X_{(k)}$ for any pair i, k, i $<\mathrm{k}, \mathrm{i}, \mathrm{k}=1, . ., n . R_{k i}$, the distance between order statistics $X_{(i)}$ and $X_{(k)}$, can range from 0 , which means both mutations are located at the same position, to $N-1$, which means the mutations are on the first and last positions of the protein sequence. Intermediate values between 0 and $N-1$ are also possible, for example $R_{k i}=1$ implies that the mutations are adjacent to each other and so on. We develop the distribution of $R_{k i}$ for each possible scenario.

$R_{k i}=0$, for any pair i, $\mathrm{k}, \mathrm{i}<\mathrm{k}, \mathrm{i}, \mathrm{k}=1, . ., n$, implies that mutations $X_{(i)}$ and $X_{(k)}$ are located at the same position. Taking the $N$ possible positions into consideration, the probability that $R_{k i}=0$ is written as

$$
\begin{aligned}
& \operatorname{Pr}\left(R_{k i}=0\right)=P\left(X_{(i)}=X_{(k)}\right)=\sum_{y=1}^{N} \operatorname{Pr}\left(X_{(i)}=X_{(k)}=y\right), \text { where } \\
& \operatorname{Pr}\left(X_{(i)}=X_{(k)}=\gamma\right) \\
& =\left\{\begin{array}{l}
\sum_{v=0}^{n-k}\left(\begin{array}{l}
n \\
n-v, v
\end{array}\right)\left(\frac{1}{N}\right)^{n-v}\left(1-\frac{1}{N}\right)^{v}, y=1 \\
\sum_{u=0}^{i-1} \sum_{v=0}^{n-k}\left(\begin{array}{l}
n \\
u, n-u-v, v
\end{array}\right)\left(\frac{y-1}{N}\right)^{u}\left(\frac{1}{N}\right)^{n-u-v}\left(1-\frac{y}{N}\right)^{v}, y=2, \ldots, N-1 \\
\sum_{u=0}^{i-1}\left(\begin{array}{l}
n \\
u, n-u
\end{array}\right)\left(\frac{1}{N}\right)^{n-u}\left(\frac{N-1}{N}\right)^{u}, y=N
\end{array}\right.
\end{aligned}
$$

The distribution is derived using the properties of order statistics. For example, when $y=X_{(i)}=X_{(k)}=1$, the first $k$ order statistics are on the first position and the remaining $n-k$ order statistics are on or above the first position. Among these $n-k$ order statistics, $v$ order statistics are located strictly above the first position, with the remaining $n-k-v$ order statistics at the first position, where $v$ can range from 0 , meaning all $n$ order statistics are on the first position, to $n-k$, indicating that all the remaining order statistics are strictly larger than the first position. A similar logic applies to $y=X_{(i)}=X$
$(k)=N$. For $1<y<N$, the distribution is derived as follows: there must be $i-1$ order statistics at position $x$, where $x \leq y$; among those $i-1$ order statistics, there are $u$ order statistics where $x<y$ and $i-1-u$ with $x=y$, where $u$ can range from 0 to $i-1$. There must be $k-i+1$ order statistics at position $x=y$. Finally, there must be $n-k$ order statistics at $x$, where $x \geq y$; among those $n-k$ order statistics, there are $v$ order statistics where $x>y$ and $n-k$ $v$ where $x=y$, where $v$ can range from 0 to $n-k$. Putting all the terms together, there are $u$ order statistics located before position $y$, with probability $(y-1) N$, where $u=0, \ldots, i-1$; there are $(k-i+1)+(i-1-u)+(n-k-v)=$ $n-u-v$ order statistics at $y$ with probability $1 / N$; there are $v$ order statistics after position $y$, with probability $1-y /$ $N$, where $v=0, \ldots, n-k$ and $x=2, \ldots, N-1$.

For $R_{k i}=1$, for any pair i, $\mathrm{k}, \mathrm{i}<\mathrm{k}, \mathrm{i}, \mathrm{k}=1, . ., \mathrm{n}$, the order statistics $X_{(i)}$ and $X_{(k)}$ are adjacent to each other. The probability distribution can be written as:

$$
\begin{aligned}
& \operatorname{Pr}\left(R_{k i}=1\right)=P\left(X_{(k)}-X_{(i)}=1\right)=\sum_{y=1}^{N-1} \operatorname{Pr}\left(X_{(i)}=y, X_{(k)}=\gamma+1\right), \text { where } \\
& \operatorname{Pr}\left(\mathrm{X}_{(i)}=\gamma, X_{(k)}=\gamma+1\right)= \\
& \left\{\begin{array}{l}
\sum_{q=0}^{k-i-1} \sum_{v=0}^{n-k}\left(\begin{array}{l}
n \\
i+q, n-i-q-v, v)
\end{array}\right)\left(\frac{1}{N}\right)^{i+q}\left(\frac{1}{N}\right)^{n-i-q-v}\left(1-\frac{2}{N}\right)^{v}, y=1 \\
\left\{\sum_{u=0}^{i-1} \sum_{q=0}^{k-i-1} \sum_{v=0}^{n-k}\left(\begin{array}{l}
n \\
u, i-u+q, n-i-q-v, v
\end{array}\right)\left(\frac{\gamma-1}{N}\right)^{u}\left(\frac{1}{N}\right)^{n-u-v}\left(1-\frac{\gamma+1}{N}\right)^{v}\right. \\
y=2, \ldots, N-1
\end{array}\right.
\end{aligned}
$$

For $R_{k i}=r$, for any pair i, $\mathrm{k}, \mathrm{i}<\mathrm{k}, \mathrm{i}, \mathrm{k}=1, . ., n, r=$ $2, \ldots, N-1$, the distribution can be written as:

$$
\begin{aligned}
& \operatorname{Pr}\left(R_{k i}=r\right)=P\left(X_{(k)}-X_{(i)}=r\right)=\sum_{\gamma=1}^{N-r} \operatorname{Pr}\left(X_{(i)}=\gamma, X_{(k)}=\gamma+r\right) \text {, where } \\
& \operatorname{Pr}\left(\mathrm{X}_{(i)}=\gamma, X_{(k)}=y+r\right)= \\
& \left.\sum_{q=0}^{k-i-1-1, k-i-1-q} \sum_{t=0}^{n-n_{v=0}} \sum_{v=k}\left\{\begin{array}{l}
n \\
i+q, t, n-i-q-v-t, v
\end{array}\right)\left(\frac{r-1}{N}\right)^{t}\left(\frac{1}{N}\right)^{n-\nu-t}\left(1-\frac{r+1}{N}\right)^{v}\right\}, y=1
\end{aligned}
$$

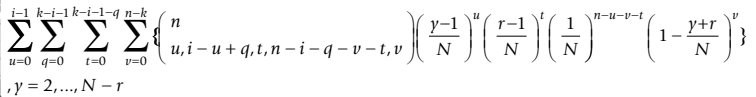

The distributions for $R_{k i}=1$ and $R_{k i}=r$ derived above, for any pair i, k, i $<\mathrm{k}, \mathrm{i}, \mathrm{k}=1, . ., n, r=2, \ldots, N-1$, is based on similar logic as $R_{k i}=0$. The $i-1$ order statistics must be located at or before position $X_{(i)}$, and the $n-k$ order statistics must be located at or after position $X_{(k)}$. For the remaining $k-i-1$ order statistics, $q$ order statistics are located at position $X_{(i)}, t$ order statistics are strictly between $X_{(i)}$ and $X_{(k)}$ and the remaining $k-i-1-q-t$ statistics are at position $X_{(k)}$, where $q=0, \ldots, k-i-1$ and $t$ $=0, \ldots, k-i-1-q$. Grouping all the terms together yields the distribution equations for $R_{k i}=1$ and $R_{k i}=r$, for any pairs of i, k, i < k, i, k = 1, .., $n, r=2, \ldots, N-1$.

Finally, for the special case of $\mathrm{i}=1$ and $\mathrm{k}=n$, the distribution of $R_{k i}$ may be simplified as 


$$
\begin{aligned}
& \operatorname{Pr}\left(R_{n 1} \leq r\right) \\
& =\left\{\begin{array}{l}
\left(\frac{1}{N}\right)^{n-1}, r=0 \\
\left(\frac{1}{N}\right)^{n-1}+\sum_{\gamma=1}^{r}(N-\gamma)\left\{\left(\frac{y+1}{N}\right)^{n}-2\left(\frac{y}{N}\right)^{n}+\left(\frac{y-1}{N}\right)^{n}\right\}, r=1, \ldots, N-1 \\
1, r \geq N
\end{array}\right.
\end{aligned}
$$

Note that $\operatorname{Pr}\left(\mathrm{R}_{n 1} \leq r\right)=1$ for $r=N-1$. The result is the same as the range statistics reported in Burr, I.W. (1955) [40].

\subsection{Approximation of the distribution}

The derivation in section 1.1 is the exact distribution of the statistical measure for nonrandom mutation clustering in the discrete uniform distribution. Proteins typically contain hundreds or thousands of amino acids and it is convenient to approximate the discrete uniform distribution with a continuous uniform distribution $(0,1)$ because calculating the distribution of $R_{k i}=r$ can be extremely slow when the length of the protein sequence $N$ or the number of mutations $n$ is large, resulting in dramatically increased iterations in those summations. For computational efficiency, we now develop the distribution for the test statistics in the continuous limit.

When the $n$ order statistics are random samples from a uniform distribution $(0,1)$, the probability distribution of order statistics $X_{(i)}$ and $X_{(k)}$, for any pair i, k, i < k, i, $\mathrm{k}=1, . .$, is:

$$
\begin{aligned}
& \operatorname{Pr}\left(R_{k i}=\frac{X(k)^{-X}(i)}{N}=r\right) \\
& =\int_{x=0}^{1-r} \frac{n !}{(i-1) !(k-i-1) !(n-k) !} x^{i-1} r^{k-i-1}(1-(x+r))^{n-k} d x
\end{aligned}
$$

where distance is normalized to be in the range $(0,1)$, so the distance $R_{k i}=\left(X_{(k)}-X_{(i)}\right) / N$ differs by the constant $N$ from section 1.1 , where $R_{k i}=X_{(k)}-X_{(i)}$. The cumulative distribution can be written as $\operatorname{Pr}\left(R_{k i} \leq r\right)$

$$
\begin{aligned}
& \operatorname{Pr}\left(R_{k i} \leq r\right) \\
& =\int_{y=0}^{r} \int_{x=0}^{1-y} \frac{n !}{(i-1) !(k-i-1) !(n-k) !} x^{i-1} y^{k-i-1}(1-(x+y))^{n-k} d x d y
\end{aligned}
$$

which by iterated integration by parts gives:

$$
\begin{aligned}
& \operatorname{Pr}\left(R_{k i} \leq r\right)=\int_{0}^{r} y^{k-i-1}(1-y)^{i+n-k} \frac{n !}{(k-i-1) !(i+n-k) !} d y \\
& =\operatorname{Pr}(\operatorname{Beta}(y ; k-i, i+n-k+1) \leq r)
\end{aligned}
$$

Using the continuous uniform distribution, $R_{k i}$ simply follows a Beta distribution with parameters $k-i$ and $i+n$ - $k+1$, ensuring that $\operatorname{Pr}\left(R_{k i} \leq 1\right)=1$. This result was reported in Johnson et al. (1995) [42] for a joint distribution of pair-wise order statistics following a continuous uniform distribution $(0,1)$.

\subsection{Correction for multiple testing}

For each pair-wise order statistic, the exact and continuous distributions can be calculated using formulas in sections 1.1 and 1.2. Clusters are evaluated for each pair of order statistics, which can elevate the false positive rate due to multiple testing. A Bonferroni correction can be chosen to correct the false positive rate because it doesn't require an independent hypotheses assumption and it is a conservative test. The false discovery rate (FDR) developed by Benjamini and Hochberg (1995) [43] is popular and has been applied to multiple testing problems in many areas. Although it requires an independent test statistics assumption, it is known to be powerful and robust under positively correlated test statistics (Benjamini and Yekutieli (2001) [44]). Because of its conservativeness, Bonferroni is applied as the default to adjust multiple testing for the NMC algorithm and as an alternative, FDR can be applied.

\subsection{NMC algorithm}

The exact and approximate distributions of distance between pair-wise order statistics were derived in section 1.1 and 1.2. The calculation is rapid for the special case when $R_{k i}$ is 0 or 1 or for the range statistics, and we use the exact distribution derived in section 1.1 to ensure accuracy for these cases. For further efficiency when calculating the distribution for $R_{k i}=1$, the algorithm is stopped when the iterated summation in the distribution reaches the significance level because the full summation is larger than the partial summation and the difference cannot be significant. The continuous distribution is used for computational efficacy when the difference $R_{k i}$ is greater than 1 . The nonrandom mutation clustering (NMC) algorithm is summarized in the following procedure:

- Input: Number and location of missense mutations in a protein

- Output: A table with columns of nonrandom mutation cluster size, starting location of the cluster, ending location of the cluster, number of mutations observed in the cluster and probability of the cluster that is significant after Bonferroni or FDR correction.

- NMC algorithm:

- Step 1: Reorder the mutation positions into order statistics and set the significance level $\alpha$. By default, $\alpha=$ 0.05 .

- Step 2: For each pair-wise order statistics, calculate the probability $\operatorname{Pr}\left(R_{k i} \leq r\right)$, for any pair i, k, i $<\mathrm{k}, \mathrm{i}, \mathrm{k}=$ $1, . ., n$. For $\mathrm{R}=0$ and 1 and/or $\mathrm{i}=1$ and $\mathrm{k}=n$, use the distribution in section 1.1. For $r>1$, use the distribution in section 1.2.

- Step 3: Calculate the Bonferroni or FDR corrected probabilities. 
- Step 4: Report the multiple-testing corrected significant clusters in the output table after sorting from the lowest probability to the highest.

The $\mathrm{R}$ source code is available in Additional file 1 and an analysis of minimum number of mutations required for NMC algorithm is available in Additional file 2.

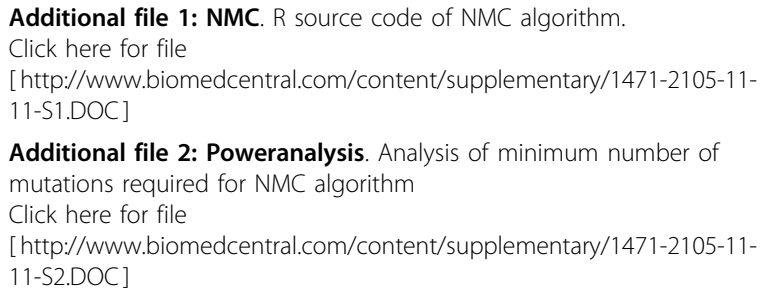

\section{Acknowledgements}

$J Y, A P, E A L$ and PAR are full-time Pfizer employees. $C T$ was a full-time Pfizer employee at the time of the work. The authors thank Professor David M. Rocke from University of California, Davis for helpful discussions and suggestions on the paper. In addition, the authors thank two anonymous referees for their insightful comments.

\section{Author details}

'Global Pre-Clinical Statistics, Pfizer Global Research and Development, 1077 Science Center Drive, San Diego, CA, 92121, USA. ${ }^{2}$ Computational Biology Group, Oncology Research Unit, Pfizer Global Research and Development, San Diego, CA, 92121, USA. ${ }^{3}$ Statistics, Corporate Analytics, Amylin Pharmaceuticals Inc, 9360 Towne Centre Drive, San Diego, CA, 92121, USA.

\section{Authors' contributions}

JY designed and developed the statistical method, and coded the NMC algorithm in R. AP and PAR proposed the idea of detecting activating mutations with nonrandom clusters. AP acquired the COSMIC database and prepared the data. JY and AP performed the analysis and drafted the manuscript. EAL and PAR contributed the idea of three-dimensional mutation detection. CT contributed the idea of the statistical method. EAL, PAR and $C T$ revised the manuscript. PAR finalized the manuscript. All authors read and approved the final manuscript.

\section{Competing interests}

The authors declare that they have no competing interests.

Received: 12 June 2009

Accepted: 7 January 2010 Published: 7 January 2010

\section{References}

1. Vogelstein B, Kinzler KW: Cancer genes and the pathways they control. Nat Med 2004, 10:789-799.

2. Weinstein IB, Joe AK: Mechanisms of disease: Oncogene addiction-a rationale for molecular targeting in cancer therapy. Nat Clin Pract Oncol 2006, 3:448-457.

3. Cahill DP, Kinzler KW, Vogelstein B, Lengauer C: Genetic instability and darwinian selection in tumours. Trends Cell Biol 1999, 9:M57-60.

4. Wang TL, Rago C, Silliman N, Ptak J, Markowitz S, Willson JKV, Parmigiani G, Kinzler KW, Vogelstein B, Velculescu VE: Prevalence of somatic alterations in the colorectal cancer cell genome. PNAS 2002, 99:3076-3080.

5. Ding L, Getz G, Wheeler DA, Mardis ER, McLellan MD, Cibulskis K, Sougnez C, Greulich H, Muzny DM, Morgan MB, Fulton L, Fulton RS, Zhang Q, Wendl MC, Lawrence MS, Larson DE, Chen K, Dooling DJ, Sabo A, Hawes AC, Shen $H$, Jhangiani SN, Lewis LR, Hall O, Zhu Y, Mathew T, Ren Y, Yao J, Scherer SE, Clerc K, Metcalf GA, Ng B, Milosavljevic A, GonzalezGaray ML, Osborne JR, Meyer R, Shi X, Tang Y, Koboldt DC, Lin L, Abbott R, Miner TL, Pohl C, Fewell G, Haipek C, Schmidt H, Dunford-Shore BH, Kraja A, Crosby SD, Sawyer CS, Vickery T, Sander S, Robinson J, Winckler W, Baldwin J, Chirieac LR, Dutt A, Fennell T, Hanna M, Johnson BE, Onofrio RC,
Thomas RK, Tonon G, Weir BA, Zhao X, Ziaugra L, Zody MC, Giordano T, Orringer MB, Roth JA, Spitz MR, Wistuba II, Ozenberger B, Good PJ, Chang AC, Beer DG, Watson MA, Ladanyi M, Broderick S, Yoshizawa A, Travis WD, Pao W, Province MA, Weinstock GM, Varmus HE, Gabriel SB, Lander ES, Gibbs RA, Meyerson M, Wilson RK: Somatic mutations affect key pathways in lung adenocarcinoma. Nature 2008, 455:1069-1075.

6. Bardelli A, Parsons DW, Silliman N, Ptak J, Szabo S, Saha S, Markowitz S, Willson JKV, Parmigiani G, Kinzler KW, Vogelstein B, Velculescul VE: Mutational analysis of the tyrosine kinome in colorectal cancers. Science 2003, 300:949.

7. Yang Z, Ro S, Rannala B: Likelihood models of somatic mutation and codon substitution in cancer genes. Genetics 2003, 165:695-705.

8. Samuels Y, Wang Z, Bardelli A, Silliman N, Ptak J, Szabo S, Yan H, Gazdar A, Powell SM, Riggins GJ, Willson JKV, Markowitz S, Kinzler KW, Vogelstein B, Velculescu1 VE: High frequency of mutations of the PIK3CA gene in human cancers. Science 2004, 304:554.

9. Davies H, Hunter C, Smith R, Stephens P, Greenman C, Bignell G, Teague J, Butler A, Edkins S, Stevens C, Parker A, O'Meara S, Avis T, Barthorpe S, Brackenbury L, Buck G, Clements J, Cole J, Dicks E, Edwards K, Forbes S, Gorton M, Gray K, Halliday K, Harrison R, Hills K, Hinton J, Jones D, Kosmidou V, Laman R, Lugg R, Menzies A, Perry J, Petty R, Raine K, Shepherd R, Small A, Solomon H, Stephens Y, Tofts C, Varian J, Webb A, West S, Widaa S, Yates A, Brasseur F, Cooper CS, Flanagan AM, Green A, Knowles M, Leung SY, Looijenga LHJ, Malkowicz B, Pierotti MA, Teh BT, Yuen ST, Lakhani SR, Easton DF, Weber BL, Goldstraw P, Nicholson AG, Wooster R, Stratton MR, Futreal PA: Somatic mutations of the protein kinase gene family in human lung cancer. Cancer Res 2005, 65:7591-7595.

10. Greenman C, Wooster R, Futreal PA, Stratton MR, Easton DF: Statistical analysis of pathogenicity of somatic mutations in cancer. Genetics 2006, 173:2187-2198

11. Sjöblom T, Jones S, Wood LD, Parsons DW, Lin J, Barber TD, Mandelker D, Leary RJ, Ptak J, Silliman N, Szabo S, Buckhaults P, Farrell C, Meeh P, Markowitz SD, Willis J, Dawson D, Willson JKV, Gazdar AF, Hartigan J, Wu L, Liu C, Parmigiani G, Park BH, Bachman KE, Papadopoulos N, Vogelstein B, Kinzler KW, Velculescu VE: The consensus coding sequences of human breast and colorectal cancers. Science 2006, 314:268-274.

12. Kaminker JS, Zhang Y, Waugh A, Haverty PM, Peters B, Sebisanovic D, Stinson J, Forrest WF, Bazan JF, Seshagiri S, Zhang Z: Distinguishing cancer-associated missense mutations from common polymorphisms. Cancer Res 2007, 67:465-473.

13. Yip YL, Zoete V, Scheib H, Michielin O: Structural assessment of single amino acid mutations: application to TP53 function. Hum Mutat 2006, 27:926-937.

14. Graur D, Li W-H: Dynamics of Genes in Population. Fundamentals of Molecular Evolution Sinauer Associates, 2 2000, 39-65.

15. Torkamani A, Schork NJ: Prediction of cancer driver mutations in protein kinases. Cancer Res 2008, 68:1675-1682.

16. Naus J: The distribution of the size of the maximum cluster of points on a line. JASA 1965, 60(310):532-538.

17. Naus Jl: A power comparison of two tests of non-random clustering. Technometrics 1966, 8:493-517.

18. Balakrishnan N, Koutras MV: Runs and scans with applications. Wiley, New York 2002.

19. Glaz J, Zhang Z: Maximum scan score-type statistics. Statistics and Probability Letters 2006, 76:1316-1322.

20. Forbes SA, Bhamra G, Bamford S, Dawson E, Kok C, Clements J, Menzies A Teague JW, Futreal PA, Stratton MR: The Catalogue of Somatic Mutations in Cancer (COSMIC). Curr Protoc Hum Genet 2008, Chapter 10(Unit 10):11.

21. Davies H, Bignell GR, Cox C, Stephens P, Edkins S, Clegg S, Teague J, Woffendin H, Garnett MJ, Bottomley W, Davis N, Dicks E, Ewing R, Floyd Y, Gray K, Hall S, Hawes R, Hughes J, Kosmidou V, Menzies A, Mould C, Parker A, Stevens C, Watt S, Hooper S, Wilson R, Jayatilake H, Gusterson BA, Cooper C, Shipley J, Hargrave D, Pritchard-Jones K, Maitland N, ChenevixTrench G, Riggins GJ, Bigner DD, Palmieri G, Cossu A, Flanagan A, Nicholson A, Ho JW, Leung SY, Yuen ST, Weber BL, Seigler HF, Darrow TL, Paterson H, Marais R, Marshall CJ, Wooster R, Stratton MR, Futreal PA: Mutations of the BRAF gene in human cancer. Nature 2002, 417:949-954

22. Bos JL: The ras gene family and human carcinogenesis. Mutat Res 1988 , 195:255-271. 
23. Hagen T, Vidal-Puig A: Characterisation of the phosphorylation of $\beta$ catenin at the GSK-3 priming site Ser45. Biochem Biophys Res Comm 2002, 294(2):324-328.

24. Morin PJ, Sparks AB, Korinek V, Barker N, Clevers H, Vogelstein B, Kinzler KW: Activation of beta-catenin-Tcf signaling in colon cancer by mutations in beta-catenin or APC. Science 1997, 275:1787-1790.

25. Fan $Y-X$, Wong L, Ding J, Spiridonov NA, Johnson RC, Johnson GR: Mutational activation of ErbB2 reveals a new protein kinase autoinhibition mechanism. J Biol Chem 2008, 283(3):1588-1596.

26. Mata IF, Wedemeyer WJ, Farrer MJ, Taylor JP, Gallo KA: LRRK2 in Parkinson's disease: protein domains and functional insights. Trends Neurosci 2006, 29:286-293.

27. Inzelberg R, Jankovic J: Are Parkinson disease patients protected from some but not all cancers?. Neurology 2007, 69:1542-1550, and discussion.

28. Strongosky AJ, Jankovic J: Are Parkinson disease patients protected from some but not all cancers?. Neurology 2008, 71:1650.

29. Nigro JM, Baker SJ, Preisinger AC, Jessup JM, Hosteller R, Cleary K, Signer SH, Davidson N, Baylin S, Devilee P, Glover T, Collins FS, Weslon A, Modali R, Harris CC, Vogelstein B: Mutations in the p53 gene occur in diverse human tumour types. Nature 1989, 342:705-708.

30. Hollstein M, Sidransky D, Vogelstein B, Harris CC: p53 mutations in human cancers. Science 1991, 253:49-53.

31. Song $H$, Hollstein $M, X u Y$ : p53 gain-of-function cancer mutants induce genetic instability by inactivating ATM. Nat Cell Biol 2007, 9:573-580.

32. Tolkacheva T, Chan AM: Inhibition of H-Ras transformation by the PTEN/ MMAC1/TEP1 tumor suppressor gene. Oncogene 2000, 19:680-689.

33. Yu WP, Pallen CJ, Tay A, Jirik FR, Brenner S, Tan YH, Venkatesh B: Conserved synteny between the fugu and human PTEN locus and the evolutionary conservation of vertebrate PTEN function. Oncogene 2001, 20:5554-5561.

34. Wagner A: Rapid detection of positive selection in genes and genomes through variation clusters. Genetics 2007, 176:2451-2463.

35. Jones S, Zhang X, Parsons DW, Lin JC-H, Leary RJ, Angenendt P, Mankoo P, Carter H, Kamiyama H, Jimeno A, Hong S-M, Fu B, Lin M-T, Calhoun ES, Kamiyama M, Walter K, Nikolskaya T, Nikolsky Y, Hartigan J, Smith DR, Hidalgo M, Leach SD, Klein AP, Jaffee EM, Goggins M, Maitra A, lacobuzioDonahue C, Eshleman JR, Kern SE, Hruban RH, Karchin R, Papadopoulos N, Parmigiani G, Vogelstein B, Velculescu VE, Kinzler KW: Core signaling pathways in human pancreatic cancers revealed by global genomic analyses. Science 2008, 321:1801-1806.

36. Parsons DW, Jones S, Zhang X, Lin JC-H, Leary RJ, Angenendt P, Mankoo P, Carter H, Siu I-M, Gallia GL, Olivi A, McLendon R, Rasheed BA, Keir S, Nikolskaya T, Nikolsky Y, Busam DA, Tekleab H, Diaz LA Jr, Hartigan J, Smith DR, Strausberg RL, Marie SKN, Shinjo SMO, Yan H, Riggins GJ, Bigner DD, Karchin R, Papadopoulos N, Parmigiani G, Vogelstein B, Velculescu VE, Kinzler KW: An integrated genomic analysis of human glioblastoma multiforme. Science 2008, 321:807-812.

37. Cancer Genome Atlas Research Network: Comprehensive genomic characterization defines human glioblastoma genes and core pathways. Nature 2008, 455:1061-1068.

38. Sved J, Bird A: The expected equilibrium of the CpG dinucleotide in vertebrate genomes under a mutation model. PNAS 1990, 87:4692-4696.

39. Jones PA, Rideout WM, Shen JC, Spruck CH, Tsai YC: Methylation, mutation and cancer. Bioessays 1992, 14:33-36.

40. Burr IW: Calculation of exact sampling distribution of ranges from a discrete population. The Annals of Mathematical Statistics 1955, 26:530-532.

41. Evans $\mathrm{DL}$, Leemis $\mathrm{LM}$, Drew JH: The distribution of order statistics for discrete random variables with applications to bootstrapping. INFORMS Journal on Computing 2006, 18(1):19-30.

42. Johnson NL, Kotz S, Balakrishnan N: Continuous Univariate Distributions. Wiley-Interscience, 2 1995, 2:280-281.

43. Benjamini $Y$, Hochberg $Y$ : Controlling the false discovery rate: a practical and powerful approach to multiple testing. JRSS, Series B 1995, 57(1):289300.

44. Benjamini $Y$, Yekutieli D: The control of the false discovery rate in multiple testing under dependency. Annals of Stat 2001, 29(4):1165-1188.

45. Berman HM, Westbrook J, Feng Z, Gilliland G, Bhat TN, Weissig H, Shindyalov IN, Bourne PE: The Protein Data Bank. Nucleic Acids Research 2000, 28:235-242.

46. Huang $\mathrm{CH}$, Mandelker D, Schmidt-Kittler O, Samuels Y, Velculescu VE, Kinzler KW, Vogelstein B, Gabelli SB, Amzel LM: The structure of a human p110alpha/p85alpha complex elucidates the effects of oncogenic PI3Kalpha mutations. Science 2007, 318:1744-1748.

47. Wang $Y$, Rosengarth $A$, Luecke $H$ : Structure of the human p53 core domain in the absence of DNA. Acta Crystallogr, Sect D 2007, 63:276-281.

doi:10.1186/1471-2105-11-11

Cite this article as: Ye et al:: Statistical method on nonrandom clustering with application to somatic mutations in cancer. BMC Bioinformatics 2010 11:11.
Publish with Bio Med Central and every scientist can read your work free of charge

"BioMed Central will be the most significant development for disseminating the results of biomedical research in our lifetime. "

Sir Paul Nurse, Cancer Research UK

Your research papers will be:

- available free of charge to the entire biomedical community

- peer reviewed and published immediately upon acceptance

- cited in PubMed and archived on PubMed Central

- yours - you keep the copyright
BioMedcentral 